
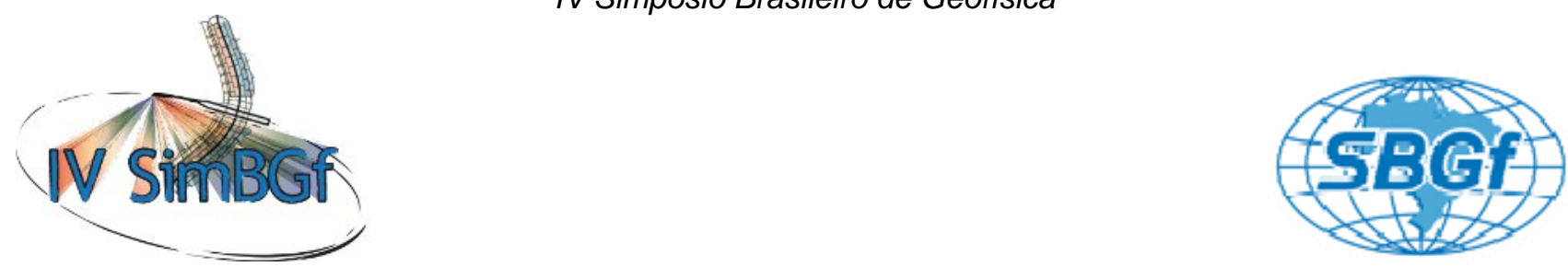

\title{
O registro morfológico de condições de regressão forçada na plataforma continental ao largo do Estado do Rio de Janeiro
}

\author{
Arantes-Oliveira, R. ${ }^{1+}$; Maia, R. M. C. ${ }^{2++}$; Malaquias; L. $^{1+++}$; Reis, A.T. ${ }^{1}$; Silva, C. G. ${ }^{2}$ \\ ${ }^{1}$ Faculdade de Oceanografia-UERJ; ${ }^{2}$ Lagemar-UFF ; ${ }^{+}$Bolsista de IC-PIBIC/CNPq; ${ }^{++}$Bolsista CAPES-Ciências do Mar; ${ }^{+++}$Bolsista de IC- \\ FAPERJ
}

Copyright 2010, SBGf - Sociedade Brasileira de Geofísica

Este texto foi preparado para a apresentação no IV Simpósio Brasileiro de Geofísica Brasília, 14 a 17 de novembro de 2010. Seu conteúdo foi revisado pelo Comitê Técnico do IV SimBGf, mas não necessariamente representa a opinião da SBGf ou de seus associados. É proibida a reprodução total ou parcial deste material para propósitos comerciais sem prévia autorização da SBGf.

\begin{abstract}
The northern shelf portion of the Santos Basin, off Rio de Janeiro State, shows a series of morphological features, such as erosional steps and scarps. Previous studies have interpreted such features as the result of erosion and sedimentation related to the Last Glacial Maximum and sea-level stillstands during the Holocene transgression. In this work, based on the interpretation of almost $5,000 \mathrm{~km}$ of single-channel seismic profiles (GEOMAR cruises), other processes are proposed to explain the formation of these features. Seismic and morphological analyses allowed the subdivision of the continental shelf off Rio de Janeiro into two main morphological sectors, whose limit is roughly defined by bathymetric contour line of approximately -100m: a proximal sector characterized by a rather smooth relief with few morphological irregularities is present up to -100 $\mathrm{m}$; while the distal deeper sector, encompassing the middle and outer shelf, is characterized by a rather irregular morphology. The morphology of the shelf proximal sector was interpreted as the result of the deposition of inner shelf sedimentary prisms formed during high sea levels, while the erosional steps presented in the distal sector are a response to forced regression conditions that occurred during the Upper Pleistocene sea level lowering that took place, according to geological data worldwide, approximately between 120 20 ky BP.
\end{abstract}

\section{Introdução}

A área de estudo se localiza na porção nordeste da bacia de Santos, abrangendo a região entre a plataforma continental interna até a região de quebra da plataforma/talude superior, imediatamente adjacente ao Estado do Rio de Janeiro e parte do Estado de São Paulo, aproximadamente entre a Ilha de São Sebastião e Cabo Frio (Fig. 1). Trata-se de uma área carente de estudos sobre o Quaternário, principalmente em função de uma escassa base de dados geológico-geofísicos. Dentre os poucos trabalhos existentes nessa região, Costa et al., (1988) identificaram irregularidades morfológicas nas proximidades das cotas batimétricas de -130, -110 e -60 m e utilizaram a comparação com outros trabalhos para a sua interpretação: a escarpa presente na cota batimétrica de -130 m estaria, de acordo com os autores, associada com sedimentos carbonáticos, que segundo Kowsmann et al. (1977) podem ser interpretadas como ambientes de praia desenvolvidos durante o Máximo Glacial; as escarpas desenvolvidas em torno de $-110 \mathrm{~m}$ foram observadas em grande parte da plataforma continental brasileira e, assim como as escarpas observadas proximas à $-60 \mathrm{~m}$, foram interpretada como estabilizações durante a trangressão holocênica (Kowsmann e Costa, 1979).

Contudo, a evolução morfológica e estratigráfica pleistocênica e holocênica da plataforma da bacia de Santos permance bastante desconhecida. Recentemente, Maia et al. (2010) propuseram um primeiro arcabouço estratigráfico Quaternário para a plataforma do Estado do Rio de Janeiro, baseado na recuperação de antigos dados sísmicos. Foi identificada uma série de sequências deposicionais dominantemente regressivas, de idade atribuída ao Pleistoceno MédioSuperior, interpretadas como depositadas nos últimos 500 ka em condições dominantemente regressivas de regressão forçada.

\section{Metodologia/ Problema Investigado}

A metodologia de trabalho consistiu basicamente em duas etapas e bases de dados: (1) a interpretação de perfis de sísmica de reflexão rasa monocanal obtidos durante as Operações GEOMAR XX e GEOMAR XVI (sismica sparker de 500-1000 J). A interpretação dos perfis sísmicos foi baseada nos conceitos de Estratigrafia Sísmica e de Seqüências, sintetizados em Catuneanu (2006); (2) a pesquisa, triagem e tratamento de dados batimétricos de sondagem derivados de folhas de bordo do Centro Hidrográfico da Marinha-CHM, e a utilização de dados de batimetria de satélite (GEBCO de 30arc segundos de resolução). Estes dados foram tratados através de mediana e gridados com o método de superfície de curvatura mínima, em seguida filtrados pelo método de semelhança máxima utilizando-se o software livre GMT (Generic Mapping Tools) para gridagem, que também permite criação dos mapas. 


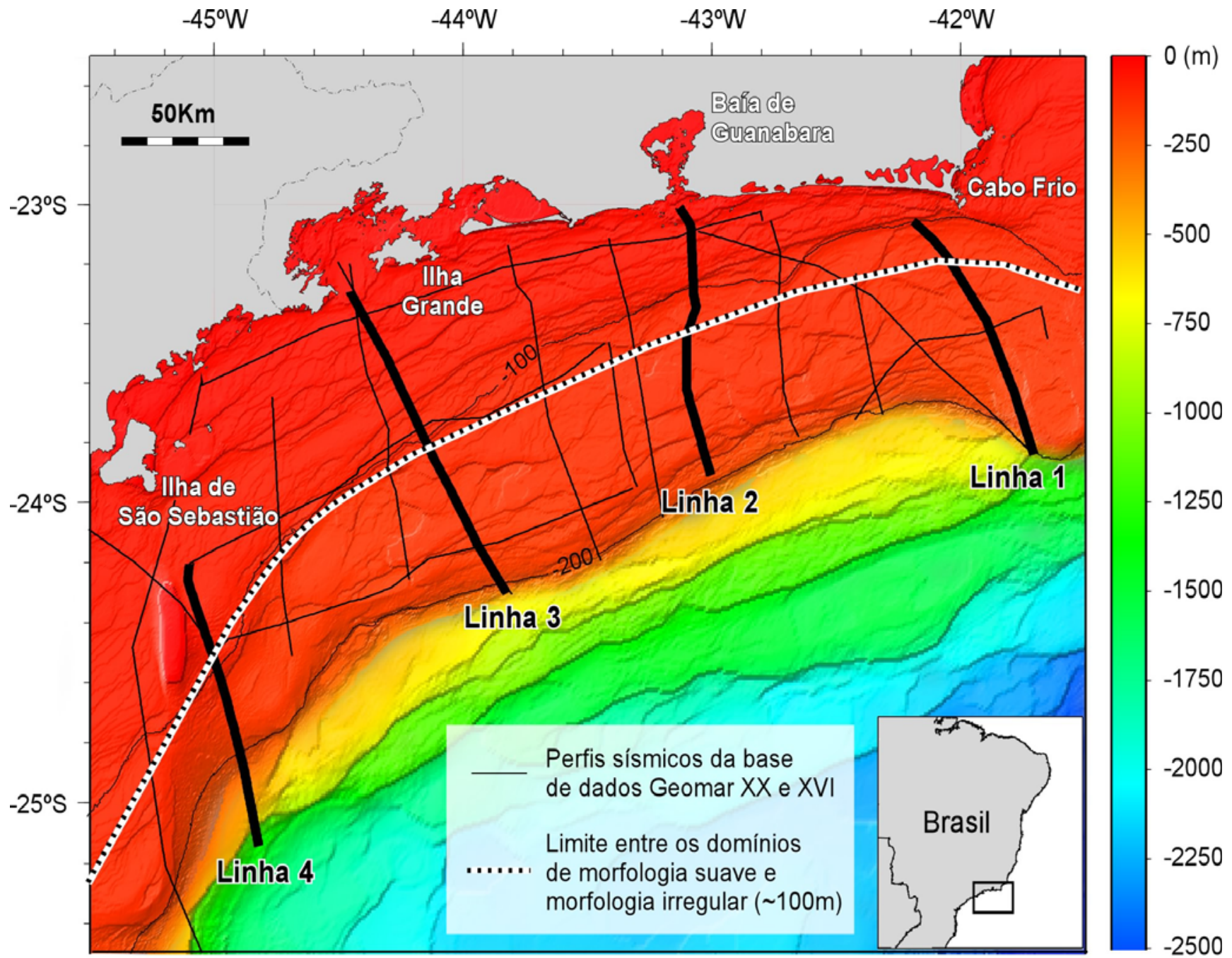

Figura 1 - Mapa batimétrico com o posicionamento dos perfis sísmicos monocanais da base de dados Geomar e do limite aproximado entre os principais domínios morfológicos da plataforma continental.

\section{Resultados}

A análise sísmica e morfológica permitiu a subdivisão da plataforma continental da área de estudos em dois setores morfológicos claramente definidos em termos da batimetria de detalhe: um setor mais raso de morfologia mais suave, englobando a plataforma interna (i); e um setor mais profundo, de morfologia residual bastante irregular, representado pela plataforma média e externa (ii) (Maia, 2009). A cota batimétrica de $-100 \mathrm{~m}$ marca regionalmente o limite aproximado entre os dois domínios (Figs. 1 e 2A).

(i) A zona morfológica proximal corresponde à região da plataforma continental interna, dominada por feições de fundo de morfologia suave e forma externa convexa, que se estendem por toda a plataforma até a cota batimétrica de -100 m (Figs. 1 e 2A). Corrêa et al. (1980) e Costa et al. (1988), observando as terminações destas feições em direção ao mar (Fig. 3A e Fig. 4C), reconheceram a existência de feições chamadas de "escarpas", às quais foram atribuídas uma origem transgressiva, desenvolvidas durante situações de estabilização de nível de mar que teriam gerado retrabalhamento e redeposição sedimentar formando cordões arenosos. A base de dados sísmicos do presente trabalho permite constatar que essas feições não podem ser consideradas escarpas, pois apresentam morfologia e características internas de prismas sedimentares de caráter essencialmente deposicional, desenvolvidas durante construção de sistemas deposicionais de mar alto (Fig. 3B, Fig. 4D e modelo esquemático em Fig. 2B). 


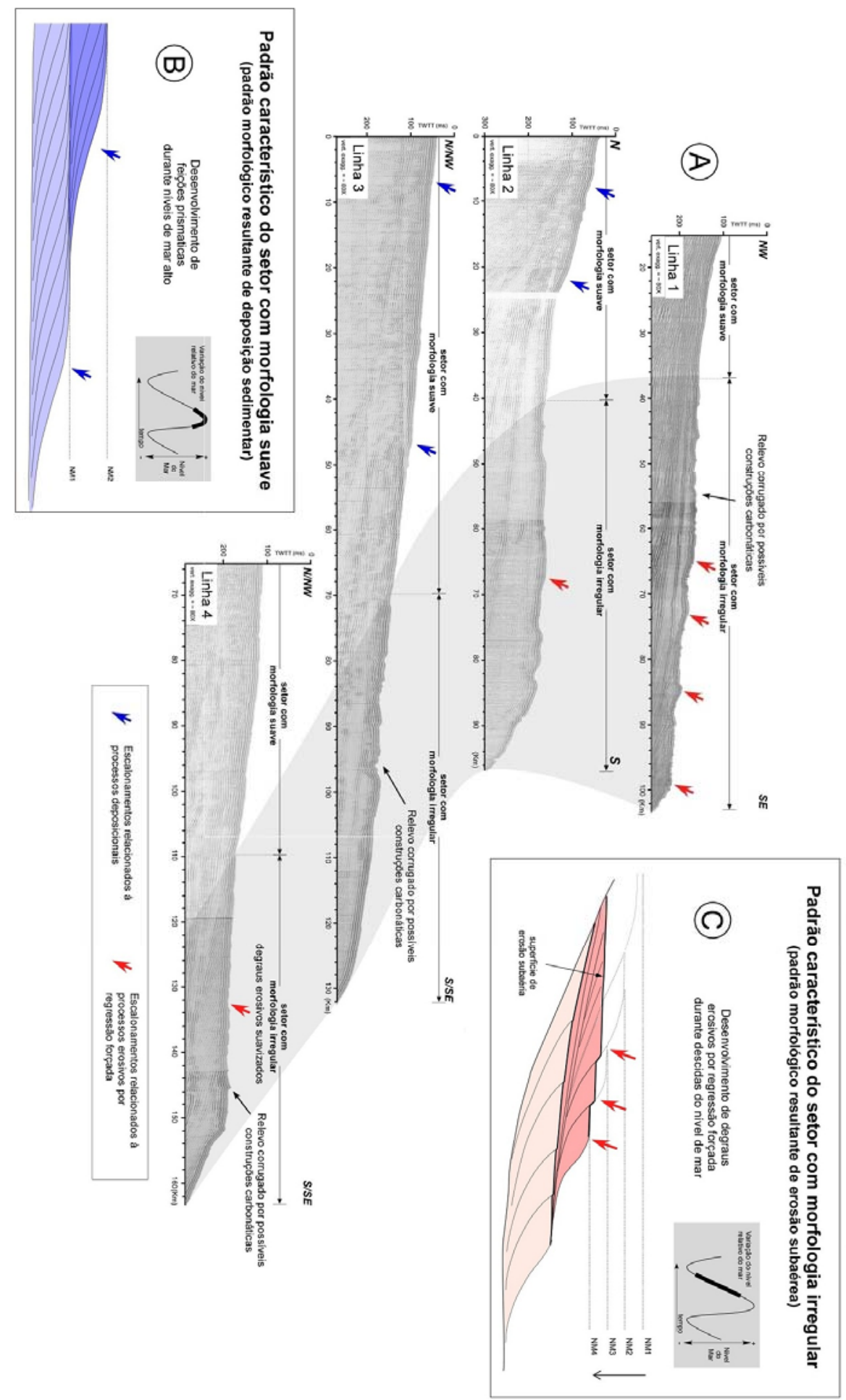

Figura 2 - A) Perfis sísmicos destacando a morfologia da plataforma continental da bacia de Santos ao largo do Estado do Rio de Janeiro (Linhas 1-4 localizadas na figura 1); B) Modelo esquemático da deposição sedimentar desenvolvida na plataforma rasa durante construção de sistemas sedimentares de mar alto, explicativo do padrão morfológico observado na plataforma continental da área de estudo, até cerca de -100 de profundidade; C) Modelo esquemático da erosão de fundo submarino, desenvolvido em condições de regressão forçada, explicativo da formação dos patamares morfológicos e das escarpas que marcam a morfologia da plataforma continental área de estudo abaixo de $-100 \mathrm{~m}$ de profundidade. 
(ii) O setor da plataforma média-externa, abaixo da cota batimétrica de $-100 \mathrm{~m}$, se caracteriza pela presença de irregularidades locais com desnível morfológico marcante, formando sucessivos escalonamentos morfológicos do fundo, limitados por escarpamentos erosivos, que formam patamares de cerca de $15-20 \mathrm{~m}$ de desnível (Figs. 1 e 2A). Os escalonamentos morfológicos do fundo submarino foram interpretados por Costa et al. (1988) como feições erosivas e sedimentares desenvolvidas no último Máximo Glacial e a Trangressão Holocênica. De acordo com Costa et al. (1988), as feições observadas em torno de $-110 \mathrm{~m}$ foram interpretadas como retrabalhamento sedimentar ocorridas em níveis de mar estacionário durante a Trangressão Holocênica (Fig. 4A), assim como também observado $\mathrm{e}$ interpretado neste trabalho (dunas transgressivas representadas na Fig. 4B).

Por outro lado, Costa et al. (1988) não atribuem uma natureza e origem clara às feições que os autores observam próximo à cota batimétrica de $-130 \mathrm{~m}$, atribuídas indistintamente à processos erosivos e/ou deposicionais desenvolvidos durante o Máximo Glacial e a Transgressão Holocênica. No presente trabalho, a maioria dos escarpamentos de fundo submarino observados abaixo da profundidade de -100 m são interpretados como remanescentes erosivos formados em condições de regressão forçada (Fig. 4B). Tais feições devem corresponder a períodos de aceleração de queda de nível eustático, e aumento da ação erosiva, resultando na impressão de uma série de patamares no fundo submarino, em posição topográfica sucessivamente mais baixa em direção à borda de plataforma (modelo esquemático em Fig. 2C). Estes patamares morfológicos apresentam ainda relevo local bastante irregular (com poucos metros de desnível), conferindo-lhes uma superfície de fundo bastante corrugada (Fig. 2A). De acordo com Simões (2007), a passagem de uma morfologia mais suave para uma morfologia irregular na plataforma da porção norte da bacia de Santos coincide com a transição entre domínios de sedimentos detríticos siliciclásticos para domínios de sedimentos carbonáticos. Na verdade, a maior parte dos estudos realizados na plataforma média a externa da área de estudos relata a presença de sedimentação carbonática abaixo da cota batimétrica de $-100 \mathrm{~m}$ ( $p$. ex. Figueiredo Jr. e Tessler, 2004). Neste sentido, as pequenas feições irregulares que se superpõem aos patamares erosivos são provavelmente de natureza bioconstrucional, devendo corresponder a construções carbonáticas que recobrem e modificam as feições erosivas impressas no fundo submarino durante as condições de regressão forçada (Fig. 2A).

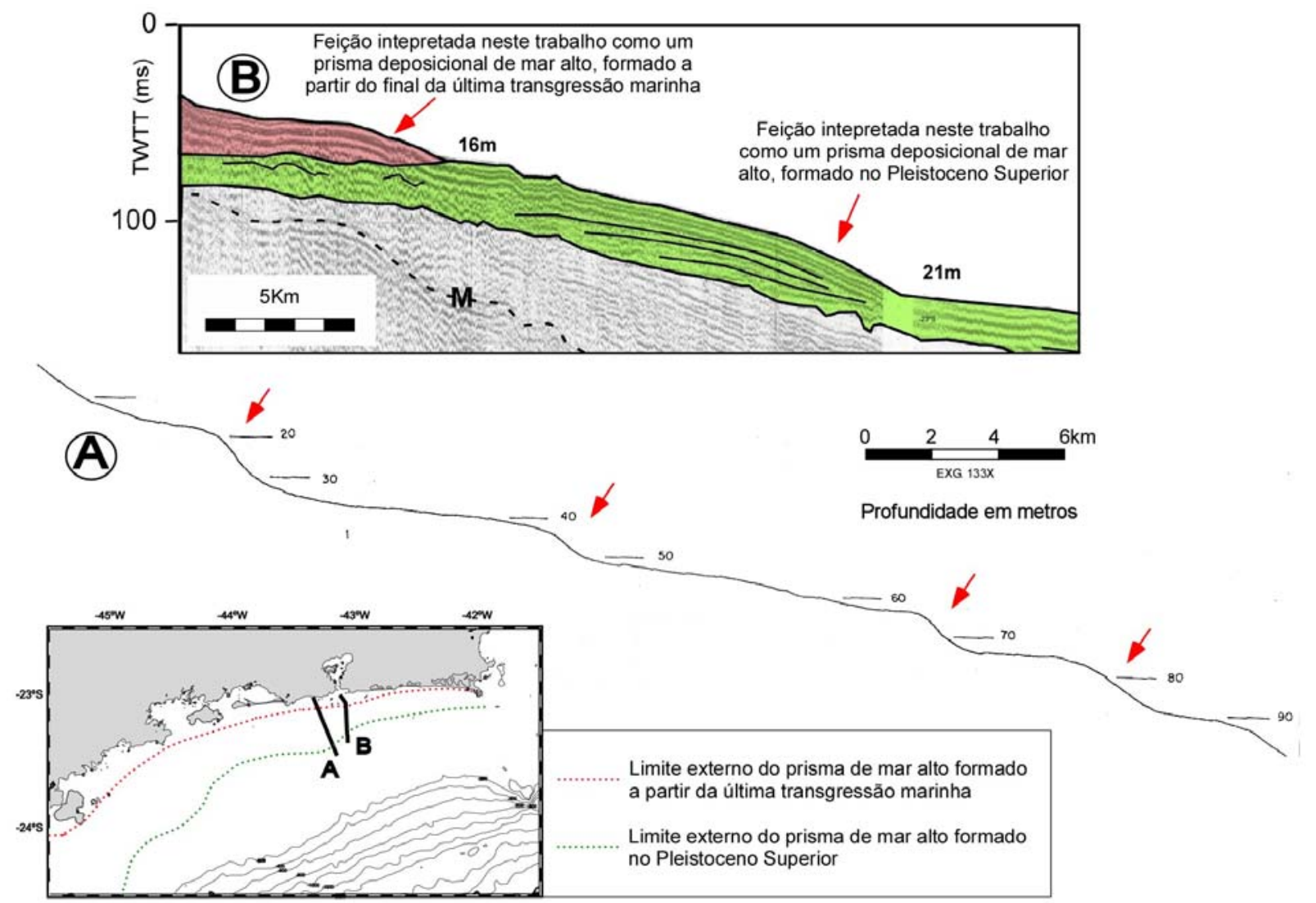

Figura 3 - Morfologia atual da plataforma continental entre Cabo Frio e Ilha de São Sebastião, entre a costa e a cota batimétrica de -100 m. (A) perfil batimétrico de Corrêa et al. (1980); (B) detalhes de feições deposicionais observadas nas linhas sísmicas do presente estudo e desenvolvidas durante os níveis de mar alto atual (em rosa) e pleistocênico superior (120 ka - em verde) As setas vermelhas indicam os desníveis batimetricos. 


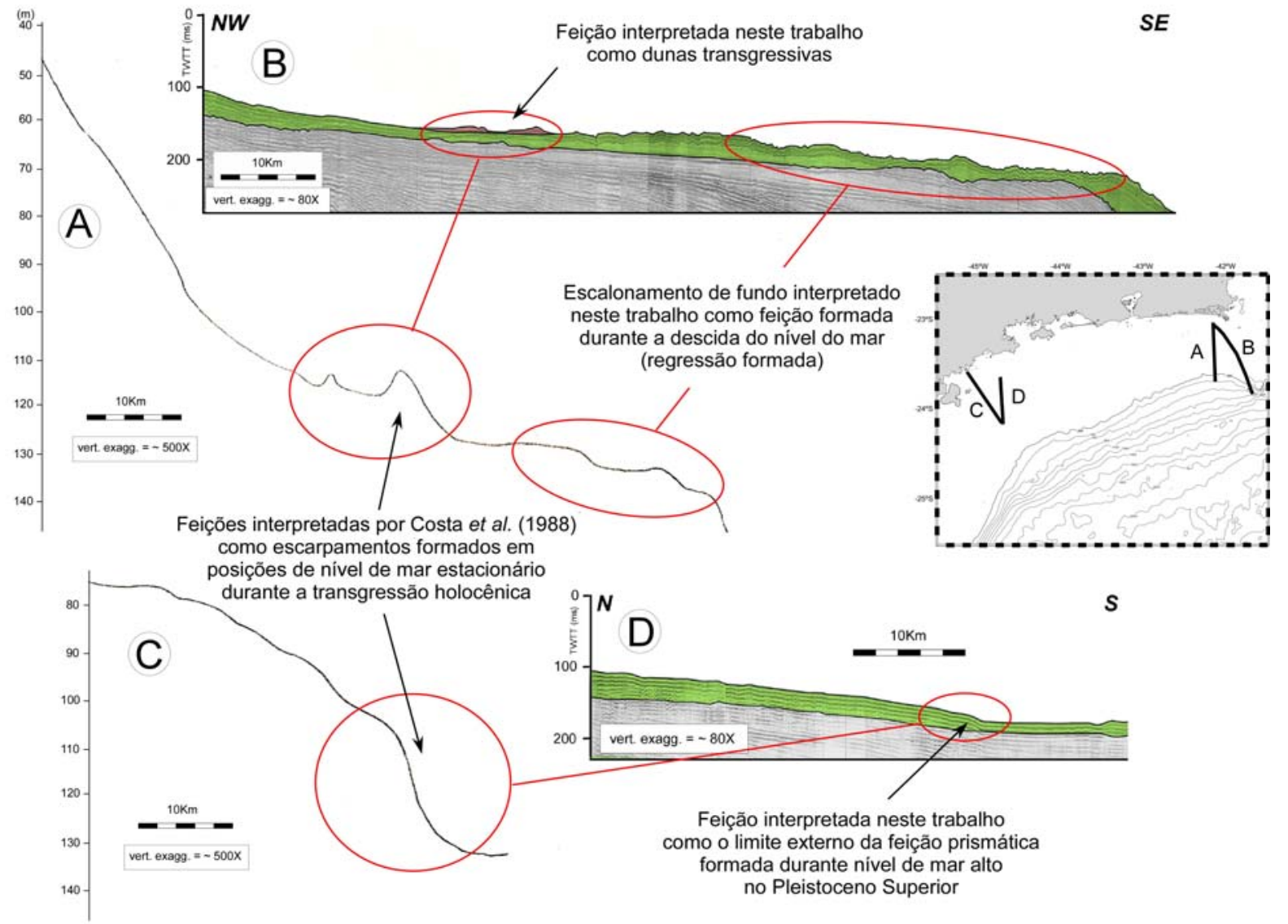

Figura 4 - Esquema comparativo relacionando a interpretação prévia disponível na literatura científica sobre algumas feições morfológicas presentes na área de estudos, e a interpretação para as mesmas feições consideradas no presente estudo. (A) e (C) correspondem a perfis batimétricos de Costa et al. (1988); (B) e (C) correspondem a detalhes de feições observadas nas linhas sísmicas do presente estudo. As feições em rosa correspondem a depósitos formados nos últimos 20 ka e as feições em verde correspondem a depósitos formados entre 120 e 20 ka.

\section{Discussão e Conclusões}

A análise sísmica empreendida no presente trabalho permitiu a subdivisão da plataforma continental da porção nordeste da bacia de Santos em duas zonas morfológicas principais: uma zona proximal até $-100 \mathrm{~m}$, marcada por uma morfologia suave decorrente da preponderância de processos deposicionais formados durante posições de nível de mar alto; e uma zona distal desde a profundidade de $-100 \mathrm{~m}$ até a borda da plataforma, marcada por escalonamentos morfológicos decorrentes de erosão ocorrida em condições de regressão forçada, implantadas durante o penúltimo ciclo glacial ocorrido entre cerca de 120 a 20 ka BP, recobertos ainda por relevo bio-construcional de origem carbonática, o que the confere grandes irregularidades locais. Deste modo, as principais feições morfológicas na escala regional da plataforma continental da bacia de Santos parecem ser impressas predominantemente pelas oscilações glacio-eustáticas ocorridas durante o Pleistoceno Superior e Holoceno.

\section{Referências}

Catuneanu, O. 2006. Principles of Sequence Stratigraphy Ed. Elsevier. 375p.

Corrêa, I. C. S.; Ponzi, V. R. A.; Trindade, L. A. F. 1980. Níveis Marinhos Quaternários da Plataforma Continental do Rio de Janeiro. XXXI Cong. Bras. Geologia. 578-587.

Costa, M. P. A.; Alves, E. C.; Pacheco, P. G.; Maia, A. S. 1988. Prováveis Estabilizações do Nível do Mar Holocênico em Trechos da Plataforma Continental entre o Norte de São Paulo e o Sul do Rio de Janeiro, Constatadas Através de Morfologia de Detalhe. XXXV Cong. Bras. Geologia. 436-450.

Figueiredo, JR, A. G. e Tessler, M. G. (2004). Topografia e composição do substrato marinho da Região SudesteSul do Brasil. São Paulo, Instituto Oceanográfico, USP. Série Documentos Revizee - Score Sul. 64p.

Maia, R. M. C.; Reis, A. T.; Alves, E. C.; Silva, C. G.; Silva, A.; Guerra, J. V.; Gorini, C.; Gorini, C.; Silva, A.; 
Arantes-Oliveira, R. (2010). Architecture and stratigraphic framework of shelf sedimentary systems off Rio de Janeiro state, northern Santos basin-Brazil. . Brazilian Journal of Oceanography, 58(special issue, IGCP526):1529, 2010.

Maia, R. M. C. 2009. Arcabouço estratigráfico e ciclicidade deposicional dos sistemas sedimentares plataformais ao largo do Estado do Rio de Janeiro-bacia de Santos. Dissertação de Mestrado - Universidade Federal Fluminense, 2009. 125 p.

Kowsmann, R. O.; Costa, M. P. A.; Vicalvi, M. A.; Coutinho, M. G. N.; Gamboa, L. A. P.. 1977. Modelo de sedimentação holocênica na plataforma continental brasileira. Série REMAC, Rio de Janeiro, v.2, p. 7-26

Kosmann, R. O. e Costa, M. P. A. 1979. Mapa faciológico dos sedimentos superficiais da plataforma, e da sedimentação quaternária no oceano profundo. Coletânea de mapas, série REMAC v.12

Simões, I. C. V. P. 2007. Aplicação da batimetria multifeixe para definição da morfologia detalhada do fundo marinho ao largo da laguna de Araruama e Arraial do Cabo - RJ. Niterói. Dissertação de Mestrado Universidade Federal Fluminense, 2007. 121 p. 